\title{
ON THE DESIGN OF TURBO TRELLIS CODED MODULATION SCHEMES USING SYMBOL-BASED EXIT CHARTS
}

\author{
S. X. $\mathrm{Ng}^{1}$, J. Kliewer ${ }^{2}$, O. R. Alamri ${ }^{1}$ and L. Hanzo ${ }^{1}$ \\ ${ }^{1}$ School of ECS, University of Southampton, SO17 1BJ, UK. \\ Email: \{sxn,ora02r,lh\}@ecs.soton.ac.uk, http://www-mobile.ecs.soton.ac.uk \\ 2 Dept. of Elec. Eng., University of Notre Dame, Notre Dame, IN 46556, U.S.A. \\ Email: jkliewer@nd.edu
}

\begin{abstract}
In this paper we design bandwidth efficient parallel-concatenated Turbo Trellis Coded Modulation (TTCM) schemes for communicating over AWGN and uncorrelated Rayleigh fading channels. The convergence properties of the symbolbased TTCM schemes employing various constituent codes, were analysed using symbol-based EXtrinsic Information Transfer (EXIT) charts. The traditional method used of generating EXIT charts is based on computationally complex multidimensional histogram measurements, which is only feasible for analysing TTCM schemes employing loworder modulation schemes, such as 4PSK and 8PSK. Hence, a novel low-complexity technique was employed in this paper for computing the symbol-based EXIT charts. Capacity-approaching TTCM schemes were designed based on the best constituent codes found when employing 8PSK, 16QAM, 32QAM and 64QAM modulation schemes.
\end{abstract}

\section{INTRODUCTION}

Trellis Coded Modulation (TCM) [1] was originally proposed for transmission over Additive White Gaussian Noise (AWGN) channels, but later it was further developed for applications in mobile communications $[2,3]$ due to its attractive bandwidth efficiency. Turbo Trellis Coded Modulation (TTCM) [4] is a more recent joint coding and modulation scheme that has a structure similar to that of the family of power-efficient binary turbo codes [5], but employs two identical parallel concatenated TCM schemes as component codes. A symbol-based turbo interleaver is used and the coded symbols of each component code are punctured alternatively for achieving a higher bandwidth efficiency as detailed in $[4,6]$. The design of the TTCM scheme in [4] was based on the search for the best component TCM codes using the 'punctured' minimal distance criterion, where the constituent TCM codes having the maximal 'punctured' minimal distance were sought. TTCM requires approximately $0.5 \mathrm{~dB}$ lower Signal-to-Noise Ratio (SNR) at a Bit Error Ratio (BER) of $10^{-4}$ than binary turbo codes when communicating using 8PSK over AWGN channels [4]. However, the performance of the TTCM schemes designed in [4]

The financial support of the EPSRC, Swindon UK and the EU under the auspices of the PHOENIX and NEWCOM projects, as well as the German Research Foundation is gratefully acknowledged. would exhibit an error floor when communicating over uncorrelated Rayleigh fading channels, if uncoded information bits exist in the constituent component codes [7]. Hence, specifically designed TTCM constituent codes are needed when communicating over Rayleigh fading channels.

EXtrinsic Information Transfer (EXIT) charts have recently emerged as a useful tool for analysing the convergence properties of iterative decoding invoked for concatenated binary coding schemes [8]. The advantage of EXIT charts is that the convergence of the entire decoding scheme can be evaluated based on the properties of the constituent codes, without performing their significantly more timeconsuming full decoding. EXIT charts are composed from the input and output characteristics of all Soft-Input SoftOutput (SISO) constituent decoders in terms of their extrinsic mutual information. The traditional method of calculating the EXIT characteristics is to generate the histogram of the decoder's extrinsic soft output, followed by a numerical integration in order to determine the extrinsic mutual information. As a result, near-capacity codes have been designed successfully designed by applying an EXIT chart based technique in [9, 10].

Non-binary (or symbol-based) EXIT charts have been introduced in [11] for analysing serially concatenated symbolbased decoders. Recently, this approach was also applied to parallel concatenated symbol-based TTCM schemes in [12]. An efficient method for generating non-binary EXIT charts from symbol-based a posteriori probabilities (APPs) was proposed in [13], which essentially represents a generalisation of the approach presented in [14] for the binary case. This technique is based on the fact that the symbol-based APPs generated at the output of a SISO decoder represent sufficient statistics for the channel observations at its input. More specifically, the average extrinsic information at the output of the decoder can be approximated by simple timeaveraging of the extrinsic (logarithmic) probabilities. As an advantage, the symbol-based extrinsic mutual information can be computed at a considerably lower complexity compared to the histogram-based approach.

In this contribution, we will employ the low-complexity symbol-based EXIT charts proposed in [13] for designing capacity approaching TTCM schemes.

\section{EXIT CHARTS OVERVIEW}

The decoding model for one of the two constituent TCM codes of the parallel concatenated TTCM scheme can be 
represented by Fig. 1, where the information symbol sequence $U$ is encoded by the constituent TCM encoder for yielding the encoded symbol sequence $X$. The sequence $X$ is transmitted over the communications channel and the received symbol sequence is denoted by $Y$. Note that the other constituent TCM decoder can be modelled by an $a$ priori channel, where the decoded and potentially errorinfested symbol sequence output by the other TCM decoder is represented by the symbol sequence $W$. More specifically, the sequence $\mathrm{W}$ can be thought of as the hypothetical channel-impaired i.e. error-prone sequence when the information sequence $U$ was transmitted over the a priori channel. Furthermore, the a priori symbol probabilities $A$ of the TCM-encoded symbols fed to the SISO decoder of Fig. 1 represent the extrinsic symbol probabilities that can be extracted from the output of the other TCM decoder. Based on both $Y$ and $A$, the SISO decoder computes both the a posteriori symbol probabilities $D$ and the extrinsic symbol probabilities $E$.

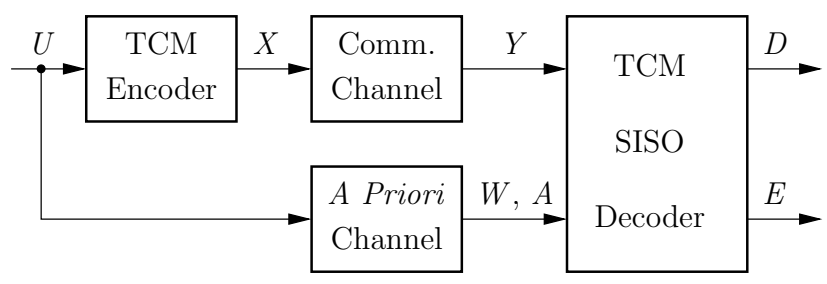

Figure 1: Decoding model for a parallel concatenated TTCM scheme.

EXIT charts visualise the input and output characteristics of the constituent SISO decoders in terms of the mutual information transfer between the uncoded input sequence $U$ and the a priori probabilities $A$ at the input, as well as between the uncoded input sequence $U$ and the extrinsic probabilities $E$ at the output of the constituent SISO decoder. We note that for binary codes the probabilities $A$ and $E$ associated with each bit are normally represented by the Log-Likelihood Ratios (LLRs) of the probability that the bit is 1 over the probability that the bit is 0 . By contrast, for non-binary codes such as TTCM, the probabilities $A$ and $E$ associated with each $m$-bit symbol are generally represented by the Log-likelihood value (L-value) for the symbol probability vector of $2^{m}$ values. Hence, there are two steps in generating an EXIT chart. Firstly, we have to model the a priori probabilities $A$ of the uncoded input sequence and then feed it to the SISO decoder. Secondly, we have to compute the mutual information between the uncoded input sequence $U$ and the extrinsic probabilities $E$ at the output of the SISO decoder.

We note that both the extrinsic and the systematic information in each a posteriori TTCM symbol probability at the output of a constituent TCM decoder cannot be separated, since the systematic and parity bits of a TTCM coded symbol are transmitted together over the communication channels $[4,6]$. However, we have to extract the extrinsic information from the a posteriori symbol probability in order to generate the EXIT chart. Hence, the assumption that both the extrinsic and systematic information are independent of each other is needed [12], so that the extrinsic information may be extracted from the a posteriori symbol probability. Nonetheless, despite the limited validity of the above-mentioned independence, we will show in Section 3.2 that accurate code design is still possible with the aid of the resultant EXIT charts.

\section{CONSTITUENT CODE SEARCH}

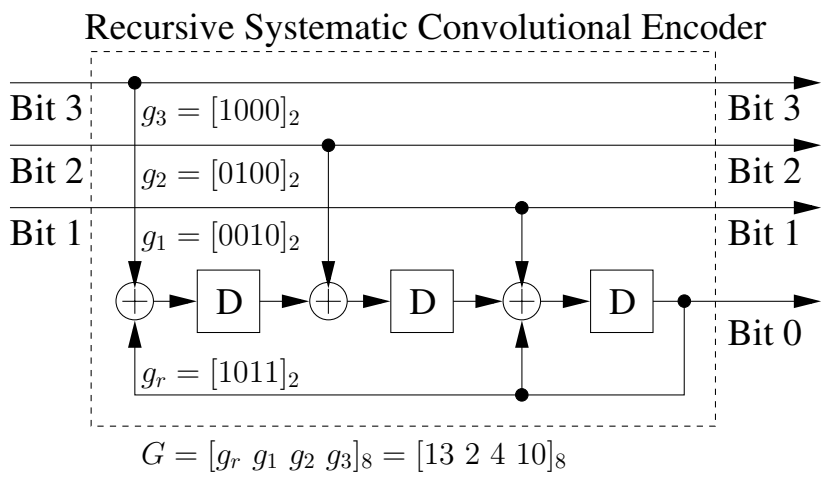

Figure 2: TCM constituent component code.

Let us first consider the encoder structure of the constituent TCM component code. Fig. 2 depicts the constituent TCM component code of an 8-state 16QAM-based TTCM scheme. The number of information bits per symbol is $m=3$ and there is only one parity bit in each TTCM coded symbol. Hence, the code rate is $R=m /(m+1)$. The connections shown in Fig. 2 between the information bits and the modulo- 2 adders are given by the generator polynomials. The feed-forward generator polynomials are denoted as $g_{i}$ for $i \in\{1,2 \ldots, m\}$, while the feed-back generator polynomial is denoted as $g_{r}$. As shown in Fig. 2, there are 4 possible connection points when there are three shift register stages, each denoted by $\mathrm{D}$. The four binary digits seen in the generator polynomials indicates the presence or absence of connections. For example, the generator polynomial corresponding to the first information bit, namely Bit 1 , is given by $g_{1}=[0010]_{2}$, which indicates that Bit 1 is connected only to the modulo- 2 adders that is third from the left. Note that we follow one of the rules in [1], where the right-most connection point is connected to the parity bit only, so that all the paths diverging from a common trellis state are associated with codewords having the same parity bit, but a least one different systematic bit [1]. The code generator is expressed in octal format as $G=\left[\begin{array}{llll}g_{r} & g_{1} & g_{2} & g_{3}\end{array}\right]_{8}=\left[\begin{array}{llll}13 & 2 & 4 & 10\end{array}\right]_{8}$.

The constituent TCM code search used for finding meritorious TTCM schemes was originally based on the 'punctured' minimal distance criterion [4]. However, we found that a constituent code having the 'punctured' maximal minimal distance guaranteed the highest coding gain only during the first turbo iteration, but it was unable to always guarantee a decoding convergence at the lowest possible SNR value. By contrast, the EXIT chart characteristics curves had the ability to predict decoding convergence, where decoding convergence is possible, when there is an open tunnel between the two EXIT chart curves [8]. Therefore, the 'punctured' maximal minimal distance is no longer the prime criterion, when designing capacityapproaching TTCM schemes. Instead, the prime design criterion is to find a constituent TCM code, where the corre- 
sponding EXIT charts exhibit an open tunnel at the lowest possible SNR value.

Since maximising the minimal distance is no longer the main design objective, we can predefine the generator polynomial connections of the information bits and only search for the best generator polynomial for the parity bit. We found that having a single one connection for each of the information bits to one distinct modulo- 2 adder, as in the feed-forward polynomials shown in Fig 2, and then searching for the best parity bit generator polynomial had the potential of providing us with constituent TCM component codes for near capacity TTCM schemes. For example, a 16QAM-based TTCM scheme employing the code shown in Fig. 2 is only $0.42 \mathrm{~dB}$ away from the channel capacity, when communicating over AWGN channels. Note that when the generator polynomials of the $m$ number of information bits are predefined as discussed above, the search space is reduced from $2^{m \nu}$ to $2^{\nu}$, where $\nu$ is the number of shift register stages. Since each information bit has a distinct connection to one modulo- 2 adder, the minimum number of shift register stages required equals the number of information bits, i.e. $\nu=m$.

\subsection{Code Search Algorithm}

We derived an algorithm for finding the TCM constituent codes using the symbol-based EXIT charts of [13], which is summarised in the flow chart shown in Fig. 3. The algorithm commences by initialising five parameters. Firstly, the connection polynomials of the $m$ information bits are initialised. Secondly, the set $G_{r}$ was constructed by storing by all the $2^{\nu}$ possible parity bit polynomials $g_{r}$. Thirdly, a step size of $\triangle=0.2 \mathrm{~dB}$ was set. Fourthly, the initial value for the SNR per information bit, namely $E_{b} / N_{0}$ was set to $\gamma=\omega+0.5 \mathrm{~dB}$, where $\omega$ is the corresponding channel capacity. Finally, the set $\Gamma$ was introduced for storing the $E_{b} / N_{0}$ values, which was initialised as a null set. Then the parity bit generator polynomial search begins by initialising the set $\mathcal{G}$ to a null set. Then the current $E_{b} / N_{0}$ value, namely $\gamma$, was assigned to the set $\Gamma$, as shown in Block 1 of Fig. 3.

The generator polynomial search loop consisting of Blocks 2, 3 and 4 is the main part of the algorithm, where the EXIT chart of each tentatively tested generator polynomial invoking a new polynomial $g_{r}$ from the full set $G_{r}$ was computed. If there is an open tunnel in its EXIT chart, then the resultant TCM code is considered a meritorious code and the corresponding $g_{r}$ value is stored in $\mathcal{G}$. The search for nearcapacity TCM codes continues until all elements in the full parity-polynomial set $G_{r}$ are tested. If none of the polynomials $g_{r}$ in the set $G_{r}$ is free from an EXIT-chart cross-over, i.e. we have $\mathcal{G}=\{\varnothing\}$, the algorithm proceeds to Block 11 . If there is a single element in $\mathcal{G}$, we have found the best TCM code and the search is concluded. However, if there are more than one elements in the set $\mathcal{G}$, we reinitialise the set $G_{r}$ using the newly found good code set $\mathcal{G}$ and proceed to Block 8. Note that we do not have to search for all possible parity bit polynomials $g_{r}$ again, when visiting the main loop (Blocks 2, 3 and 4) this time, since $G_{r}$ consists of parity bit polynomials during from the previous search which are capable of approaching the achievable capacity.

The operations represented by Blocks 8,9 and 10 are now used for reducing the $E_{b} / N_{0}$ value $\gamma$, by the stepsize $\triangle$. Note that if $(\gamma-\triangle)$ was found to be in the set $\Gamma$,

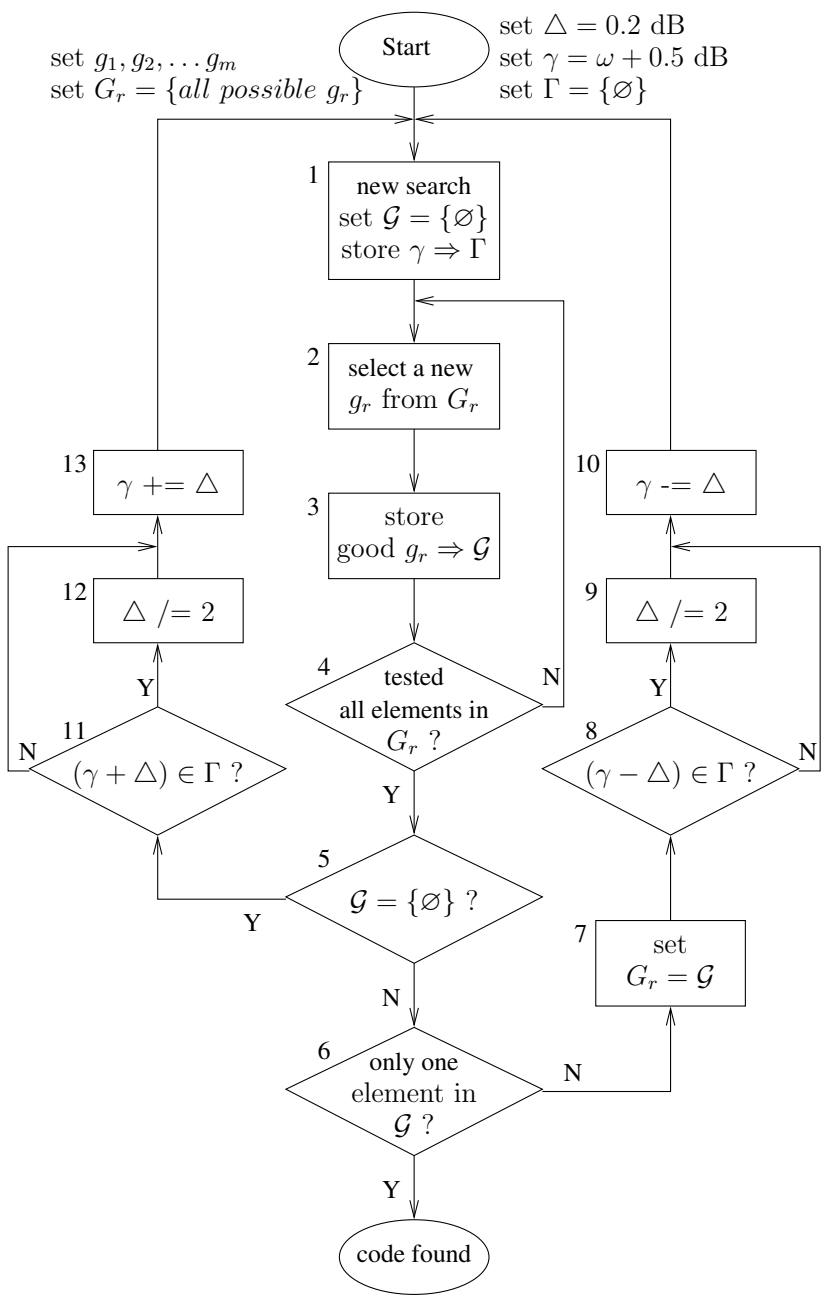

Figure 3: Code search algorithm.

this implies that we have already carried out the search based on this particular $(\gamma-\triangle)$ value before. In this case, the stepsize $\triangle$ will be halved, as shown in Block 9 , before the current $\gamma$ value is reduced by $\triangle \mathrm{dB}$. The appropriate counterpart operations are carried out in Blocks 11, 12 and 13 , where the $E_{b} / N_{0}$ value $\gamma$ is increased by the stepsize $\triangle$, when no polynomial was found in the set $\mathcal{G}$. Again, the step size will be halved, if necessary in order to avoid repeating the same search.

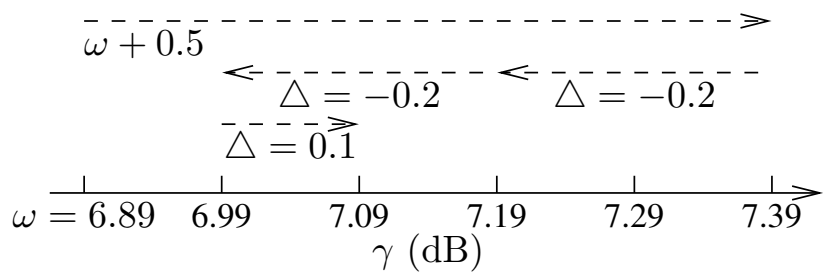

Figure 4: One-dimensional search space.

Fig. 4 illustrates the operational steps, when searching for the constituent TCM code generator polynomials for the 16-state 32QAM-based TTCM scheme. More specifically, 
the associated channel capacity is given by $\omega=6.89 \mathrm{~dB}$. Hence, according to the fourth initialling parameters, the TCM parity generator polynomial search commences at $\gamma=$ $\omega+0.5=7.39 \mathrm{~dB}$. In the example considered in Fig. 4, it takes two $\triangle=0.2 \mathrm{~dB}$ steps to the left and $1 \triangle=0.1 \mathrm{~dB}$ step to the right, before finding the best TCM parity bit polynomial, where the estimated minimum SNR required for achieving decoding convergence is $E_{b} / N_{0}=7.09 \mathrm{~dB}$. Hence, the constituent TCM code search for constructing capacity-approaching TTCM schemes consists of a number of consecutive EXIT chart evaluations and a search in a one-dimensional continuous space.

Note that, a TTCM scheme could also employ two nonidentical constituent TCM component codes. In that case, the code search algorithm depicted in Fig. 3 may be employed for matching the EXIT chart curve of one constituent TCM code to that of the other. However, in this paper we only consider classic TTCM schemes employing two identical constituent TCM codes.

\subsection{Results and Discussions}

The EXIT charts and the corresponding decoding trajectories of the TTCM schemes employing 8PSK, 16QAM and 32QAM signal sets are shown in Figs. 5, 6 and 7, respectively, when communicating over AWGN channels. As mentioned in Section 2, the EXIT charts were generated based on the assumption that the extrinsic information and the systematic information are independent of each other, which is not always true. Hence, full simulations based on actual TTCM decoding were also performed to verify the exact performance of the codes found using the proposed EXIT chart design. As can be seen from Figs. 5, 6 and 7, there are some mismatches between the EXIT charts and the simulation-based decoding trajectories.

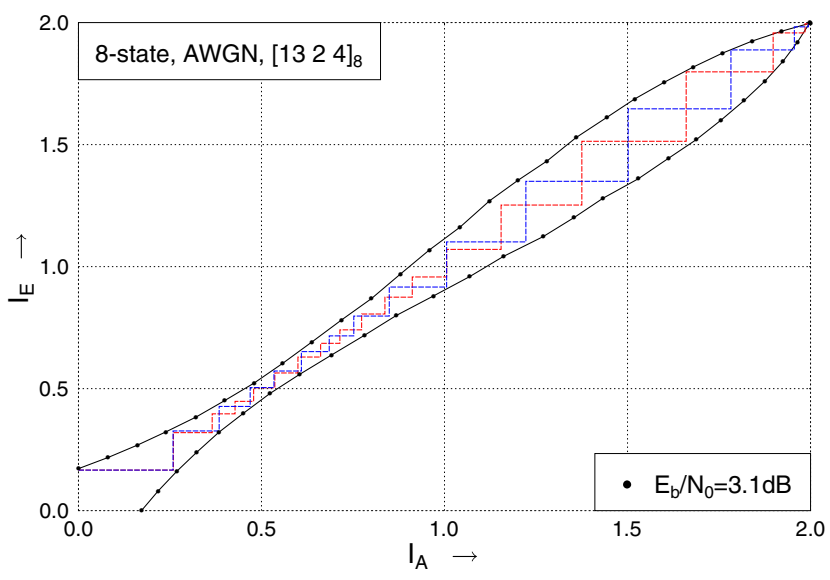

Figure 5: EXIT chart for the 8PSK-TTCM scheme and two snapshot decoding trajectories for transmission over AWGN channels using a block-length of 100000 symbols, $m=2$, 8state rate- $2 / 3$ TCM codes).

Similar to the findings of [12], where multi-dimensional histogram based EXIT charts were used, we found that the 8-state TTCM scheme employing the generator polynomial $\left[\begin{array}{lll}13 & 2 & 4\end{array}\right]_{8}$ outperforms that using $\left[\begin{array}{lll}11 & 2 & 4\end{array}\right]_{8}$. In fact, this code is only $(3.10-2.75)=0.35 \mathrm{~dB}$ away from the channel capacity, where $2.75 \mathrm{~dB}$ is the capacity limit of the $8 \mathrm{PSK}$

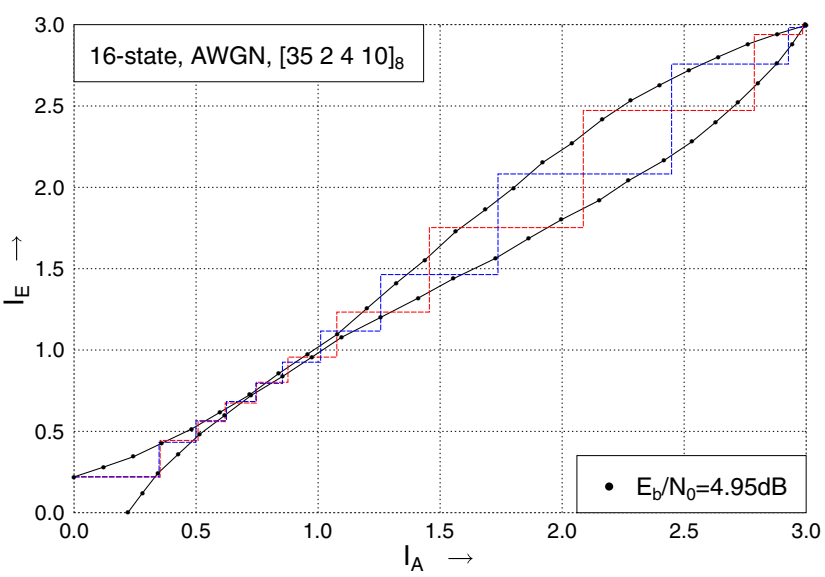

Figure 6: EXIT chart for the 16QAM-TTCM scheme and two snapshot decoding trajectories for transmission over AWGN channels using a block-length of 100000 symbols, $m=3,16$-state rate- $3 / 4$ TCM codes).

signal set in AWGN channels when the effective throughput is $2 \mathrm{bit} / \mathrm{s} / \mathrm{Hz}$ [6]. Note that the channel capacity may be further approached, if non-identical TCM component codes are employed in the TTCM scheme. For example, the performance of the 16-state 16QAM-based TTCM scheme characterised in Fig. 6 is limited by the narrow bottle neck at $I_{A} \approx 0.75$ due to using identical component codes. Hence, two different component codes having their EXIT charts carefully matched to each other would perform nearer to the channel capacity.

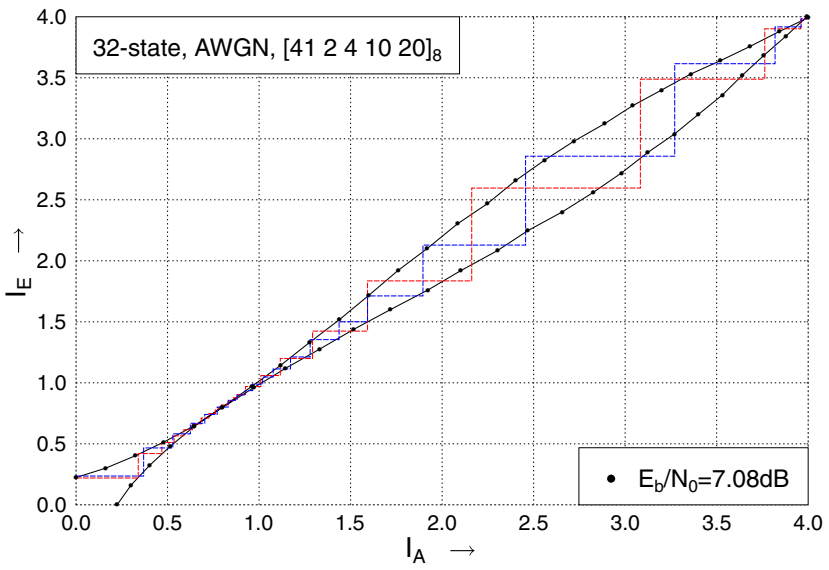

Figure 7: EXIT chart for the 32QAM-TTCM scheme and two snapshot decoding trajectories for transmission over AWGN channels using a block-length of 100000 symbols, $m=4,32$-state rate- $4 / 5$ TCM codes).

The TCM constituent codes found for transmission over both AWGN and uncorrelated Rayleigh fading channels are tabulated in Tables 1 and 2, respectively, for 8PSK, 16QAM, 32QAM and 64QAM signal sets. The EXIT chart based estimation and the simulations based $E_{b} / N_{0}$ threshold values marking the edge of the waterfall region were tabulated and compared to the channel capacity limits, $\omega$, in the tables. It was found that most of the codes designed for 


\begin{tabular}{|c|c|c|c|c|}
\hline \multirow{2}{*}{$\begin{array}{l}\text { Modulation/ } \\
\text { States }\end{array}$} & \multirow{2}{*}{$\begin{array}{l}\text { Polynomial, Octal } \\
{\left[\begin{array}{lllll}g_{r} & g_{1} & g_{2} & g_{3} & \ldots\end{array}\right]}\end{array}$} & \multicolumn{3}{|c|}{ Thresholds, dB } \\
\hline & & Est. & Actual & $\omega$ \\
\hline 8PSK $/ 4$ & {$\left[\begin{array}{lll}7 & 2 & 4\end{array}\right]$} & 3.25 & $\overline{3.40}$ & 2.75 \\
\hline $8 \mathrm{PSK} / 8$ & {$\left[\begin{array}{lll}13 & 2 & 4\end{array}\right]$} & 3.00 & 3.10 & \\
\hline 16QAM/8 & {$\left[\begin{array}{llll}13 & 2 & 4 & 10\end{array}\right]^{*}$} & 5.07 & 4.95 & 4.53 \\
\hline 16QAM/16 & {$\left[\begin{array}{llll}35 & 2 & 4 & 10\end{array}\right]^{*}$} & 4.95 & 4.93 & \\
\hline $32 \mathrm{QAM} / 16$ & {$\left[\begin{array}{lllll}37 & 2 & 4 & 10 & 20\end{array}\right]^{*}$} & 7.09 & 7.08 & 6.89 \\
\hline $32 \mathrm{QAM} / 32$ & {$\left[\begin{array}{llllll}41 & 2 & 4 & 10 & 20\end{array}\right]^{*}$} & 7.08 & 7.08 & \\
\hline 64QAM/32 & {$\left[\begin{array}{llllll}41 & 2 & 4 & 10 & 20 & 40\end{array}\right]^{*}$} & 9.65 & 9.60 & 9.15 \\
\hline 64QAM/64 & {$\left[\begin{array}{llllll}111 & 2 & 4 & 10 & 20 & 40\end{array}\right]$} & 9.65 & 9.70 & \\
\hline
\end{tabular}

Table 1: TTCM code polynomials for AWGN channels. The polynomials marked with ${ }^{*}$ yields a performance less than $0.5 \mathrm{~dB}$ away from the channel capacity.

\begin{tabular}{|c|c|c|c|c|}
\hline \multirow{2}{*}{$\begin{array}{l}\text { Modulation/ } \\
\text { States }\end{array}$} & \multirow{2}{*}{$\begin{array}{l}\text { Polynomial, Octal } \\
{\left[\begin{array}{lllll}g_{r} & g_{1} & g_{2} & g_{3} & \ldots\end{array}\right]}\end{array}$} & \multicolumn{3}{|c|}{ Thresholds, dB } \\
\hline & & Est. & Actual & $\omega$ \\
\hline 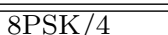 & $\mid \overline{\mid 724 \pi}$ & $\overline{c 6.80}$ & $\overline{7.58}$ & $\overline{c 5.38}$ \\
\hline $8 \mathrm{PSK} / 8$ & $\left.\mid \begin{array}{lll}13 & 2 & 4\end{array}\right]^{*}$ & 5.71 & 6.20 & \\
\hline 16QAM/8 & {$\left[\begin{array}{llll}15 & 2 & 4 & 10\end{array}\right]$} & 8.60 & 8.70 & 7.57 \\
\hline 16QAM/16 & {$\left[\begin{array}{llll}27 & 2 & 4 & 10\end{array}\right]^{*}$} & 8.06 & 8.16 & \\
\hline $32 \mathrm{QAM} / 16$ & {$\left[\begin{array}{lllll}37 & 2 & 4 & 10 & 20\end{array}\right]$} & 10.70 & 11.00 & 9.98 \\
\hline 32QAM/32 & $\left.\begin{array}{llllll}61 & 2 & 4 & 10 & 20\end{array}\right|^{*}$ & 10.55 & 10.75 & \\
\hline 64QAM/32 & {$\left[\begin{array}{llllll}67 & 2 & 4 & 10 & 20 & 40\end{array}\right]^{*}$} & 13.40 & 13.38 & 12.71 \\
\hline 64QAM/64 & {$\left[\begin{array}{lllllll}10 & 2 & 4 & 10 & 20 & 40\end{array}\right]^{*}$} & 13.27 & 13.38 & \\
\hline
\end{tabular}

Table 2: TTCM code polynomials for uncorrelated Rayleigh fading channels. The polynomials marked with * yields a performance less than $1.0 \mathrm{~dB}$ away from the channel capacity.

AWGN and uncorrelated Rayleigh fading perform within about $0.5 \mathrm{~dB}$ and $1.0 \mathrm{~dB}$ of the channel capacity, respectively. This demonstrates the efficiency of the EXIT chart based code-search algorithm proposed in Section 3.1.

\section{CONCLUSIONS}

We have designed capacity-approaching TTCM schemes by performing a search for good constituent TCM component codes with the aid of symbol-based EXIT charts. The prime design criterion of capacity-approaching TTCM schemes is that of finding an open tunnel in the corresponding EXIT charts at the lowest possible SNR values, rather than maximising the 'punctured' minimal distance of the constituent codes [4]. Hence, we can reduce the code search space by fixing the feed-forward generator polynomials and then search for the best feed-back generator polynomial that provides an open tunnel in the EXIT chart at the lowest possible SNR value. Although the assumption of extrinsic information and systematic information is not always satisfied in the symbol-based TTCM scheme, most of the good constituent codes found assist the TTCM schemes in performing near the channel capacity. Our future work will be focused on designing irregular TTCM schemes, where nonidentical component codes are employed.

\section{REFERENCES}

[1] G. Ungerböck, "Channel Coding with Multilevel/Phase Signals," IEEE Transactions on Information Theory, vol. 28, pp. 55-67, January 1982.

[2] D. Divsalar and M. K. Simon, "The design of trellis coded MPSK for fading channel: Performance criteria," IEEE Transactions on Communications, vol. 36, pp. 1004-1012, September 1988.
[3] D. Divsalar and M. K. Simon, "The design of trellis coded MPSK for fading channel: Set partitioning for optimum code design," IEEE Transactions on Communications, vol. 36, pp. 1013-1021, September 1988.

[4] P. Robertson, T. Wörz, "Bandwidth-efficient turbo trelliscoded modulation using punctured component codes," IEEE Journal on Selected Areas in Communications, vol. 16, pp. 206-218, February 1998.

[5] C. Berrou, A. Glavieux and P. Thitimajshima, "Near Shannon Limit Error-Correcting Coding and Decoding : Turbo Codes," in Proceedings, IEEE International Conference on Communications, pp. 1064-1070, 1993.

[6] L. Hanzo, S. X. Ng, W. Webb and T.Keller, Quadrature Amplitude Modulation: From Basics to Adaptive TrellisCoded, Turbo-Equalised and Space-Time Coded OFDM, $C D M A$ and $M C-C D M A$ Systems, Second Edition. New York, USA : John Wiley and Sons, 2004.

[7] S. X. Ng, T. H. Liew, L-L. Yang and L. Hanzo, "Comparative Study of TCM, TTCM, BICM and BICM-ID schemes," IEEE Vehicular Technology Conference, pp. 2450-2454, May 2001.

[8] S. ten Brink, "Convergence behaviour of iteratively decoded parallel concatenated codes," IEEE Transactions on Communications, vol. 49, pp. 1727-1737, October 2001.

[9] S. ten Brink, "Rate one-half code for approaching the Shannon limit by $0.1 \mathrm{~dB}, "$ IEE Electronics Letters, vol. 36, pp. 1293-1294, July 2000.

[10] M. Tüchler and J. Hagenauer, "Exit charts and irregular codes," in Proceeding of the 36th Annual Conference on Information and System Sciences, (Princeton, NJ, USA), March 2002.

[11] A. Grant, "Convergence of non-binary iterative decoding," in Proceedings of the IEEE Global Telecommunications Conference (GLOBECOM), (San Antonio TX, USA), pp. 1058-1062, November 2001.

[12] H. Chen and A. Haimovich, "EXIT charts for turbo trelliscoded modulation," IEEE Communications Letters, vol. 8, pp. 668-670, November 2004.

[13] J. Kliewer, S. X. Ng and L. Hanzo, "On the computation of EXIT characteristics for symbol-based iterative decoding," in 4th International Symposium on Turbo Codes in connection with 6th International ITG-Conference on Source and Channel Coding, (Munich, Germany), 3-7 April 2006.

[14] I. Land, P. Hoeher, and S. Gligorević, "Computation of symbol-wise mutual information in transmission systems with $\log$ APP decoders and application to EXIT charts," in Proc. International ITG Conference on Source and Channel Coding (SCC), (Erlangen, Germany), pp. 195-202, Jan. 2004 